\title{
Synthesis of dehydrodipeptide esters and their evaluation as inhibitors of cathepsin $\mathrm{C}$
}

\author{
Maciej Makowski $^{1} \cdot$ Paweł Lenartowicz $^{1} \cdot$ Bartosz Oszywa $^{1}$. \\ Michał Jewgiński $^{2}$. Małgorzata Pawełczak ${ }^{1}$ Paweł Kafarski ${ }^{1,2}$
}

Received: 7 October 2014/Accepted: 27 February 2015/Published online: 16 April 2015

(C) The Author(s) 2015. This article is published with open access at Springerlink.com

\begin{abstract}
The procedures for the synthesis of esters of dehydropeptides containing C-terminal (Z)-dehydrophenylalanine and dehydroalanine have been elaborated. These esters appeared to be moderate or weak inhibitors of cathepsin C, with some of them exhibiting slow-binding behavior. As shown by molecular modeling, they are rather bound at the surface of the enzyme and are not submersed in its binding cavities.
\end{abstract}

Keywords Dehydropeptides · Esterification ·

Enzyme inhibitors $\cdot$ Molecular modeling

\section{Introduction}

$\alpha, \beta$-Dehydroaminoacids present in proteins contribute to catalytic action in tyrosine aminomutase (Christenson et al., 2003) and to properties of green fluorescent proteins (Zimmer, 2002). They are also constituents of a variety of peptidic allelochemicals of microbial origin, including antimicrobial lantibiotics (nisin, subtilin, epidermin and gallidermin) (Willey and van der Donk, 2007), neurotoxins (roquefortine, oxaline and phomopsins) (Overy et al.,

Electronic supplementary material The online version of this article (doi:10.1007/s00044-015-1366-0) contains supplementary material, which is available to authorized users.

Maciej Makowski

maciej.makowski@uni.opole.pl

1 Faculty of Chemistry, Opole University, Oleska 48, 45-052 Opole, Poland

2 Department of Bioorganic Chemistry, Faculty of Chemistry, Wroclaw University of Technology, Wybrzeze Wyspianskiego 27, 50-370 Wroclaw, Poland
2005; Battilani et al., 2011), hepatotoxins (microcystins and nodularins) (Gulledge et al., 2002), phytotoxins (tentoxin and AM toxins) (Andre and Pinet, 1997; Jingfeng et al., 2013) and antitumor agents (phenylahistin) (Kanoh et al., 1999). This is because of both, the reactivity of their side-chain double bonds (especially toward thiols) (Ferreira et al., 2001; Seebeck et al., 2011) and of the ability to undertake specific forms of three-dimensional structure [they could be considered as foldamers (Goldman et al., 2007)]. The latter properties cause the growing interest in this class of compounds.

Although from some years we have been engaged in studies on the dependence of three-dimensional structure of dehydropeptides on their inhibitory activity toward cathepsin C, no clear structure-activity relationship could be drawn (Makowski et al., 2001; Latajka et al., 2006, 2008). In this paper, we present synthesis of esters of glycyl ${ }^{z}$ dehydrophenylalanine $\left(\mathrm{Gly}-{ }^{\mathrm{Z}} \Delta \mathrm{Phe}\right)$, glycyldehydroalanine (Gly$\Delta$ Ala) and L-phenylalanyldehydroalanine (Phe- $\Delta$ Ala) and evaluation of their action toward this enzyme.

\section{Materials and methods}

\section{General}

All reagents and solvents were purchased from SigmaAldrich, Avantor Performance Materials or Merck. Ethyl acetate (EtOAc), dichloromethane (DCM), diethyl ether $\left(\mathrm{Et}_{2} \mathrm{O}\right)$ and tetrahydrofuran (THF) were dried over $\mathrm{P}_{2} \mathrm{O}_{5}$ and then distilled. $N, N$-dimethyformamide (DMF) was distilled under reduced pressure and stored over molecular sieves 4 A. Other chemicals were used without purification. Reaction progress was monitored by TLC on Merck 60 silica plates. The spots were visualized by placing 
chromatogram plate at chlorine vapor followed by spraying with $o$-tolidine in water/acetic acid mixture. NMR spectra were recorded on Bruker Ultrashield $400 \mathrm{MHz}$ instrument, operating at $400 \mathrm{MHz}\left({ }^{1} \mathrm{H}\right)$ and $100 \mathrm{MHz}\left({ }^{13} \mathrm{C}\right)$. Samples were prepared in DMSO-d6 (99.8\% at. D). Chemical shifts are reported in ppm relative to TMS used as internal standard or to the signal of solvent $\left({ }^{1} \mathrm{H}\right.$ NMR $2.5 \mathrm{ppm} ;{ }^{13} \mathrm{C}$ NMR 39.52 ppm for DMSO-d6), and coupling constant is reported in Hertz. In the description of dipeptide ${ }^{1} \mathrm{H}$ NMR and ${ }^{13} \mathrm{C}$ NMR spectra, the tosylate group is omitted for better readability (Tos ${ }^{1} \mathrm{H}$ NMR (DMSO, $400 \mathrm{MHz}$ ): $\delta$ $7.48(\mathrm{~d}, J=8.0 \mathrm{~Hz}, 2 \mathrm{H}, \operatorname{Ar} \boldsymbol{H}), 7.11(\mathrm{~d}, J=8.0 \mathrm{~Hz}, 2 \mathrm{H}$, $\mathrm{Ar} \boldsymbol{H}), 2.29$ (s, 3H, $\left.\mathrm{CH}_{3}\right) ;{ }^{13} \mathrm{C}$ NMR (DMSO, $100 \mathrm{MHz}$ ): $\delta$ $20.84\left(\mathrm{CH}_{3}\right), 125.57,128.14,137.80,145.71(4 \times \mathrm{ArC})$. The copies of all NMR spectra are available at electronic supplementary material. Melting points were determined on a Stuart SMP30 apparatus and are reported uncorrected. Mass spectra were recorded on Bruker micrOTOF-Q II high-resolution mass spectrometer with electrospray ionization (ESI). IR spectra were recorded on Nicolet 6700 FT-IR spectrophotometer (Thermo Scientific) operating at resolution $2 \mathrm{~cm}^{-1}$ and scanning range $4000-400 \mathrm{~cm}^{-1}$. Samples were measured as $\mathrm{KBr}$ disks.

\section{Synthesis of $N$-protected dehydrodipeptides}

Boc-protected dehydrodipeptides containing C-terminal dehydroalanine $(\Delta \mathrm{Ala})$ or $(\mathrm{Z})$-dehydrophenylalanine $\left(\Delta^{\mathrm{Z}-}\right.$ Phe) were synthesized earlier by condensation of appropriate carboxamides with $\boldsymbol{\alpha}$-keto acids in benzene in the presence of $p$-toluenesulfonic acid as catalyst (Makowski et al., 1985).

\section{Synthesis of dehydrodipeptide methyl, ethyl and isopropyl esters}

Syntheses were based on procedure of Cossec et al. (2008). Thus, Boc-Gly- $\Delta$ Ala or Boc- $(S)$ Phe- $\Delta$ Ala was dissolved in methanol ( 0.2 or $0.4 \mathrm{M}$, respectively), and 0.5 equivalent of $\mathrm{Cs}_{2} \mathrm{CO}_{3}$ was added. The mixture was stirred for $1 \mathrm{~h}$ at room temperature followed by evaporation of solvent. The dipeptide cesium salt was dissolved in DMF (0.28 M), and fivefold or fourfold excess (respectively) of methyl, ethyl or isopropyl iodide was added in portions. After completion of the reaction (3-5 h, controlled by TLC), solvent was evaporated under reduced pressure. The obtained residue was dissolved in ethyl acetate and washed subsequently with: $1 \mathrm{M} \mathrm{HCl}$, saturated $\mathrm{KHCO}_{3}, 0.1 \mathrm{M} \mathrm{Na}_{2} \mathrm{~S}_{2} \mathrm{O}_{3}$ and brine (each one in triplicate). Organic layer was dried over anhydrous $\mathrm{MgSO}_{4}$. Product was crystallized from mixtures of diethyl ether/hexane or ethyl acetate/hexane providing Boc-Gly- $\Delta$ Ala-OMe in $91 \%$, Boc-(S)Phe- $\Delta$ Ala-
OMe in $94 \%$, Boc-(S)Phe- $\Delta$ Ala-OEt in $94 \%$ and Boc(S)Phe- $\Delta$ Ala-OPr ${ }^{\mathrm{i}}$ in $81 \%$ yields. Deprotection of amine group was performed in $20 \%$ solution of TFA in DCM. Deprotection of amine group of dehydrodipeptide esters containing dehydroalanine required the use of anisole (3\% $\mathrm{v} / \mathrm{v})$ for protection against oligomerization reactions. Mixture was stirred at room temperature for $30 \mathrm{~min}$, and equivalent of $p$-toluenesulfonic acid was added. Mixing was continued for $15 \mathrm{~min}$, and solvent was removed under reduced pressure. The residue was dissolved in dichloromethane, and solvent was carefully evaporated to remove the excess of trifluoroacetic acid. Products were crystallized from mixtures of isopropanol/hexane.

Gly-AAla-OMe.Tos $87 \%$ yield (deprotection); $\mathrm{mp}=$ 151.5-155 ${ }^{\circ} \mathrm{C}$ with decomposition; ${ }^{1} \mathrm{H}$ NMR $\delta 9.92(\mathrm{~s}, 1 \mathrm{H}$, $\mathrm{NH}), 8.02\left(\mathrm{~s}, 3 \mathrm{H}, \mathrm{NH}_{3}^{+}\right), 6.32\left(\mathrm{~s}, 1 \mathrm{H}, \mathrm{CH}_{\boldsymbol{A}} \mathrm{H}_{\mathrm{B}} \Delta_{\mathrm{Ala}}\right), 5.84(\mathrm{~s}$, $\left.1 \mathrm{H}, \mathrm{CH}_{\mathrm{A}} \boldsymbol{H}_{\boldsymbol{B}}{ }_{\Delta \mathrm{Ala}}\right), 3.80\left(\mathrm{~s}, 2 \mathrm{H}, \mathrm{CH}_{2 \mathrm{Gly}}\right), 3.78(\mathrm{~s}, 3 \mathrm{H}$, $\left.\mathrm{OCH}_{3}\right) .{ }^{13} \mathrm{C}$ NMR $\delta 166.14\left(\boldsymbol{C}=\mathrm{O}_{\text {amid. }}\right), 163.45\left(\boldsymbol{C}=\mathrm{O}_{\text {est. }}\right)$, $132.02(\boldsymbol{C}=), \quad 110.58 \quad\left(\boldsymbol{C} \mathrm{H}_{2}=\right), \quad 52.88 \quad\left(\mathrm{OCH}_{3}\right), 41.10$ $\left(\boldsymbol{C H}_{2 \mathrm{Gly}}\right)$. HRMS (ESI) $\mathrm{m} / \mathrm{z}$ calcd for $\mathrm{C}_{6} \mathrm{H}_{11} \mathrm{~N}_{2} \mathrm{O}_{3}$ $(\mathrm{M}+\mathrm{H})^{+}$159.0764; found 159.0767; IR $\left(\mathrm{KBr}, \mathrm{cm}^{-1}\right)$ 3700-2600 broad (H-bonding), $1733\left(\mathrm{C}=\mathrm{O}_{\text {ester }}\right), 1689$ IAB $\left(\mathrm{C}=\mathrm{O}_{\mathrm{amid}}\right), 1634(\mathrm{C}=\mathrm{C}), 1551$ IIAB $(\mathrm{C}-\mathrm{N}$ and $\mathrm{N}-\mathrm{H})$, 1200-1171 (C-O-C and $\left.\mathrm{SO}_{3}\right), 919\left(=\mathrm{CH}_{2}\right)$.

(S)Phe- $\triangle$ Ala-OMe.Tos $98 \%$ yield (deprotection); $\mathrm{mp}=$ 156-157 ${ }^{\circ} \mathrm{C}$ with decomposition; ${ }^{1} \mathrm{H}$ NMR $\delta 9.93$ (s, $1 \mathrm{H}$, $\mathrm{NH}), 8.25$ (s, 3H, NH$H_{3}^{+}$), 7.37-7.23 (m, 5H, ArH $\left.\boldsymbol{H}_{P \text { he }}\right), 6.27$ $\left(\mathrm{s}, 1 \mathrm{H}, \mathrm{CH}_{\boldsymbol{A}} \mathrm{H}_{\mathrm{B}}{ }_{\mathrm{AAla}}\right), 5.85\left(\mathrm{~s}, 1 \mathrm{H}, \mathrm{CH}_{\mathrm{A}} \boldsymbol{H}_{\boldsymbol{B}} \Delta \mathrm{Ala}\right), 4.42-4.34$ (m, 1H, CH $\boldsymbol{H}_{\mathrm{Phe}}$ ), 3.76 (s, 3H, OCH $\boldsymbol{H}_{3}$ ), 3.09 (ABX system, $J$ 13.9, $6.1 \mathrm{~Hz}, 1 \mathrm{H}, \mathrm{CH}_{\boldsymbol{A}} \mathrm{H}_{\mathrm{B}} \mathrm{Phe}$ ), 2.99 (ABX system, $J$ 13.9, $7.8 \mathrm{~Hz}, 1 \mathrm{H}, \mathrm{CH}_{\mathrm{A}} \boldsymbol{H}_{\boldsymbol{B}}$ Phe $) .{ }^{13} \mathrm{C} \mathrm{NMR} \delta 168.13\left(\boldsymbol{C}=\mathrm{O}_{\text {amid. }}\right)$, $163.37\left(\boldsymbol{C}=\mathrm{O}_{\text {est. }}\right), 134.60\left(\boldsymbol{C A r}_{\mathrm{Phe}}\right), 131.92(\boldsymbol{C}=), 129.58$, 128.60, $127.32\left(3 \times \boldsymbol{C A r}_{\mathrm{Phe}}\right), \quad 111.52 \quad\left(\boldsymbol{C H}_{2}=\right), \quad 53.72$ $\left(\boldsymbol{C} \mathrm{H}_{\mathrm{Phe}}\right), 52.86\left(\mathrm{OCH}_{3}\right), 37.10\left(\boldsymbol{C H}_{2 \mathrm{Phe}}\right)$. HRMS (ESI) $\mathrm{m} / \mathrm{z}$ calcd for $\mathrm{C}_{13} \mathrm{H}_{17} \mathrm{~N}_{2} \mathrm{O}_{3}(\mathrm{M}+\mathrm{H})^{+}$249.1234; found 249.1223; IR ( $\mathrm{KBr}, \mathrm{cm}^{-1}$ ) 3700-2700 broad (H-bonding), $1728\left(\mathrm{C}=\mathrm{O}_{\text {ester }}\right), 1694 \mathrm{IAB}\left(\mathrm{C}=\mathrm{O}_{\text {amid }}\right), 1638(\mathrm{C}=\mathrm{C}), 1538$ IIAB (C-N and N-H), 1203-1166 (C-O-C and $\left.\mathrm{SO}_{3}\right), 919$ $\left(=\mathrm{CH}_{2}\right)$.

(S)Phe-AAla-OEt-Tos $85 \%$ yield (deprotection); mp = 139-141 ${ }^{\circ} \mathrm{C}$; ${ }^{1} \mathrm{H}$ NMR $\delta 9.91(\mathrm{~s}, 1 \mathrm{H}, \mathrm{NH}), 8.24(\mathrm{~s}, 3 \mathrm{H}$, $\left.\mathrm{NH}_{3}^{+}\right), 7.37-7.24\left(\mathrm{~m}, 5 \mathrm{H}, \mathrm{Ar} \boldsymbol{H}_{\mathrm{Phe}}\right), 6.27\left(\mathrm{~s}, 1 \mathrm{H}, \mathrm{CH}_{\boldsymbol{A}} \mathrm{H}_{\mathrm{B}}\right.$ $\Delta \mathrm{Ala}), 5.84\left(\mathrm{~s}, 1 \mathrm{H}, \mathrm{CH}_{\mathrm{A}} \mathrm{H}_{\mathrm{B}} \Delta_{\mathrm{Ala}}\right), 4.44-4.35$ (m, 1H, $\left.\mathrm{CH}_{\mathrm{Phe}}\right)$, $4.22\left(\mathrm{q}, J=7.1 \mathrm{~Hz}, 2 \mathrm{H}, \mathrm{OCH}_{2} \mathrm{CH}_{3}\right), 3.10$ (ABX system, $J=13.9,6.2 \mathrm{~Hz}, 1 \mathrm{H}, \mathrm{CH}_{\boldsymbol{A}} \mathrm{H}_{\mathrm{B}}$ Phe), 2.99 (ABX system, $J=13.9,7.8 \mathrm{~Hz}, 1 \mathrm{H}, \mathrm{CH}_{\mathrm{A}} \boldsymbol{H}_{\boldsymbol{B}}$ Phe $), 1.25(\mathrm{t}, J=7.1 \mathrm{~Hz}$, $\left.3 \mathrm{H}, \mathrm{OCH}_{2} \mathrm{CH}_{3}\right) .{ }^{13} \mathrm{C} \mathrm{NMR} \delta 168.10\left(\boldsymbol{C}=\mathrm{O}_{\text {amid. }}\right), 162.89$ $\left(\boldsymbol{C}=\mathrm{O}_{\text {est }}\right), 134.60\left(\boldsymbol{C A r}_{\mathrm{Phe}}\right), 132.10(\boldsymbol{C}=), 129.55,128.60$, $127.31\left(3 \times \boldsymbol{C A r}_{\mathrm{Phe}}\right), 111.19\left(\boldsymbol{C} \mathrm{H}_{2}=\right), 61.74\left(\boldsymbol{C H}_{2} \mathrm{CH}_{3}\right)$, $53.70\left(\boldsymbol{C H}_{\mathrm{Phe}}\right), 37.10\left(\boldsymbol{C H}_{2 \mathrm{Phe}}\right), 13.99\left(\mathrm{CH}_{2} \boldsymbol{C H}_{3}\right)$. HRMS (ESI) $\mathrm{m} / \mathrm{z}$ calcd for $\mathrm{C}_{14} \mathrm{H}_{19} \mathrm{~N}_{2} \mathrm{O}_{3}(\mathrm{M}+\mathrm{H})^{+}$263.1390; 
found 263.1395; IR $\left(\mathrm{KBr}, \mathrm{cm}^{-1}\right)$ 3700-2450 broad (Hbonding $), 1713\left(\mathrm{C}=\mathrm{O}_{\text {ester }}\right), 1691$ IAB $\left(\mathrm{C}=\mathrm{O}_{\mathrm{amid}}\right), 1640$ $(\mathrm{C}=\mathrm{C}), 1535$ IIAB $(\mathrm{C}-\mathrm{N}$ and $\mathrm{N}-\mathrm{H}), 1249-1167(\mathrm{C}-\mathrm{O}-\mathrm{C}$ and $\left.\mathrm{SO}_{3}\right), 915\left(=\mathrm{CH}_{2}\right)$.

(S)Phe-AAla-OPr ${ }^{\mathrm{i}}$ Tos $80 \%$ yield (deprotection); $\mathrm{mp}=$ 153-155 ${ }^{\circ} \mathrm{C}$ with decomposition; ${ }^{1} \mathrm{H}$ NMR $\delta 9.88$ (s, $1 \mathrm{H}$, $\mathrm{NH}), 8.24$ (s, 3H, $\left.\boldsymbol{N H}_{3}^{+}\right), 7.39-7.21$ (m, 5H, $\left.\mathrm{Ar} \boldsymbol{H}_{\mathrm{Phe}}\right), 6.25$ (s, $\left.1 \mathrm{H}, \boldsymbol{C H}_{\boldsymbol{A}} \mathrm{H}_{\mathrm{B}} \Delta \mathrm{Ala}\right), 5.81$ (s, $1 \mathrm{H}, \mathrm{CH}_{\mathrm{A}} \boldsymbol{H}_{\boldsymbol{B}} \Delta \mathrm{Ala}$ ), 5.00 (hept, $\left.\mathrm{J}=6.2 \mathrm{~Hz}, 1 \mathrm{H}, \mathbf{C} \boldsymbol{H}\left(\mathrm{CH}_{3}\right)_{2}\right), 4.43-4.34\left(\mathrm{~m}, 1 \mathrm{H}, \mathbf{C} \boldsymbol{H}_{\text {Phe }}\right)$, $3.10\left(\mathrm{dd}, \mathrm{J}=13.9,6.1 \mathrm{~Hz}, 1 \mathrm{H}, \mathrm{ABX}\right.$ system $\mathrm{CH}_{\boldsymbol{A}} \mathrm{H}_{\mathrm{B}}$ Phe), $2.99\left(\mathrm{dd}, \mathrm{J}=13.9,7.8 \mathrm{~Hz}, 1 \mathrm{H}, \mathrm{ABX}\right.$ system $\mathrm{CH}_{\mathrm{A}} \boldsymbol{H}_{\boldsymbol{B}}$ Phe), $1.26\left(\mathrm{~d}, \mathrm{~J}=6.2 \mathrm{~Hz}, 6 \mathrm{H}, \mathrm{CH}\left(\mathrm{CH}_{3}\right)_{2}\right) \cdot{ }^{13} \mathrm{C}$ NMR $\delta 168.07$ $\left(\boldsymbol{C}=\mathrm{O}_{\text {amid. }}\right), 162.44\left(\boldsymbol{C}=\mathrm{O}_{\text {est. }}\right), 134.63\left(\boldsymbol{C}\right.$ Ar $\left._{\text {Phe }}\right), 132.34$ $(\boldsymbol{C}=), \quad 129.55, \quad 128.60, \quad 127.31 \quad\left(3 \times \boldsymbol{C A r}_{\text {Phe }}\right), \quad 110.91$ $\left(\boldsymbol{C} \mathrm{H}_{2}=\right), 69.51\left(\boldsymbol{C H}\left(\mathrm{CH}_{3}\right)_{2}\right), 53.70\left(\boldsymbol{C H}_{\mathrm{Phe}}\right), 37.11\left(\boldsymbol{C H}_{2 \mathrm{Phe}}\right)$, $21.43\left(\mathrm{CH}\left(\mathrm{CH}_{3}\right)_{2}\right)$. HRMS (ESI) $\mathrm{m} / \mathrm{z}$ calcd for $\mathrm{C}_{15} \mathrm{H}_{21} \mathrm{~N}_{2} \mathrm{O}_{3}$ $(\mathrm{M}+\mathrm{H})^{+}$277.1547; found 277.1545; IR $\left(\mathrm{KBr}, \mathrm{cm}^{-1}\right)$ 3700-2450 broad (H-bonding), $1710\left(\mathrm{C}=\mathrm{O}_{\text {ester }}\right), 1690 \mathrm{IAB}$ $\left(\mathrm{C}=\mathrm{O}_{\text {amid }}\right), 1640(\mathrm{C}=\mathrm{C}), 1534$ IIAB $(\mathrm{C}-\mathrm{N}$ and $\mathrm{N}-\mathrm{H})$, 1226-1169 (C-O-C and $\left.\mathrm{SO}_{3}\right), 919\left(=\mathrm{CH}_{2}\right)$.

\section{Synthesis of allyl and propargyl esters of dipeptides containing dehydroalanine}

A $\mathrm{Cs}_{2} \mathrm{CO}_{3} 0.163 \mathrm{~g}(0.5 \mathrm{mmol})$ was added to solution of Boc-Gly- $\Delta$ Ala $0.244 \mathrm{~g}$ ( $1 \mathrm{mmol})$ or Boc-(S)Phe- $\Delta$ Ala $0.334 \mathrm{~g}(1 \mathrm{mmol})$ in $5 \mathrm{~mL}$ of methanol. The mixture was stirred at room temperature for $2 \mathrm{~h}$, and solvent was removed under reduced pressure. Solid residue was dissolved in $5 \mathrm{~mL}$ of THF for Boc-Gly- $\Delta$ Ala or $5 \mathrm{~mL}$ of DMF for Boc-(S)Phe- $\Delta$ Ala, and allyl bromide $0.856 \mathrm{~mL}(10 \mathrm{mmol})$ or propargyl bromide $1.114 \mathrm{~mL}(10 \mathrm{mmol})$ was added dropwise over $15 \mathrm{~min}$. When peptide substrate was consumed (controlled by TLC), the solvent and excess of bromide were removed under reduced pressure. The residue was dissolved in $80 \mathrm{~mL}$ of ethyl acetate, filtrated and washed with: $1 \mathrm{M} \mathrm{HCl}(4 \times 5 \mathrm{~mL})$, saturated $\mathrm{KHCO}_{3}$ $(4 \times 5 \mathrm{~mL})$ and brine. Organic layer was dried over $\mathrm{MgSO}_{4}$ and filtered, and $0.2 \mathrm{~mL}$ of anisole was added. The solvent was removed under reduced pressure at $35^{\circ} \mathrm{C}$. The residue was dissolved in $10 \mathrm{~mL}$ DCM, $1.5 \mathrm{~mL}$ of TFA was added and the mixture was stirred for $1 \mathrm{~h}$ at room temperature followed by addition of $0.190 \mathrm{~g}(1 \mathrm{mmol})$ of $p$ toluenesulfonic acid. Stirring was continued for additional $20 \mathrm{~min}$, and solvent was removed under reduced pressure. The residue was evaporated two times with $20 \mathrm{~mL}$ of DCM to remove TFA excess. Products were crystallized from mixtures of isopropanol/hexane

Gly-AAla-OAll-Tos $72 \%$ global yield; $\mathrm{mp}=159$ $161.5{ }^{\circ} \mathrm{C}$ with decomposition; ${ }^{1} \mathrm{H}$ NMR $\delta 9.92(\mathrm{~s}, 1 \mathrm{H}, \mathrm{NH})$, $8.04\left(\mathrm{~s}, 3 \mathrm{H}, \mathrm{NH}_{3}^{+}\right), 6.34\left(\mathrm{~s}, 1 \mathrm{H}, \mathrm{CH}_{\boldsymbol{A}} \mathrm{H}_{\mathrm{B}} \Delta \mathrm{Ala}\right), 6.05-5.92$ $\left(\mathrm{m}, 1 \mathrm{H}, \mathrm{CH}_{2}=\mathrm{CH}\right), 5.88\left(\mathrm{~s}, 1 \mathrm{H}, \mathrm{CH}_{\mathrm{A}} \boldsymbol{H}_{\boldsymbol{B}} \Delta \mathrm{Ala}\right), 5.40-5.33$ $\left(2 \times \mathrm{m}, \quad 1 \mathrm{H}, \quad \mathrm{CH}=\mathrm{CH}_{\boldsymbol{A}} \mathrm{H}_{\mathrm{B}}\right), \quad 5.30-5.25 \quad(2 \times \mathrm{m}, \quad 1 \mathrm{H}$, $\left.\mathrm{CH}=\mathrm{CH}_{\mathrm{A}} \mathbf{H}_{\mathbf{B}}\right), 4.73\left(\mathrm{~m}, 2 \mathrm{H}, \mathrm{OCH}_{2}\right), 3.81\left(\mathrm{~s}, 2 \mathrm{H}, \mathrm{CH}_{2 \mathrm{Gly}}\right)$. ${ }^{13} \mathrm{C}$ NMR $\delta 166.11\left(\boldsymbol{C}=\mathrm{O}_{\text {amid. }}\right), 162.63\left(\boldsymbol{C}=\mathrm{O}_{\text {est. }}\right), 132.09$ $\left(\boldsymbol{C H}={ }_{\mathrm{All}}\right), \quad 132.01 \quad(\boldsymbol{C}=), \quad 118.37 \quad\left(\boldsymbol{C} \mathrm{H}_{2}={ }_{\mathrm{All}}\right), \quad 110.75$ $\left(\boldsymbol{C H}_{2}={ }_{\Delta \mathrm{Ala}}\right), 65.92\left(\mathrm{OCH}_{2}\right), 41.08\left(\boldsymbol{C H}_{2 \mathrm{Gly}}\right)$. HRMS (ESI) $\mathrm{m} / \mathrm{z}$ calcd for $\mathrm{C}_{8} \mathrm{H}_{13} \mathrm{~N}_{2} \mathrm{O}_{3}(\mathrm{M}+\mathrm{H})^{+} 185.0921$; found 185.0919. IR ( $\mathrm{KBr}, \mathrm{cm}^{-1}$ ) 3600-2600 broad (H-bonding), $1718\left(\mathrm{C}=\mathrm{O}_{\text {ester }}\right), 1692$ IAB $\left(\mathrm{C}=\mathrm{O}_{\text {amid }}\right), 1649(\mathrm{C}=\mathrm{C}), 1538$ IIAB $(\mathrm{C}-\mathrm{N}$ and $\mathrm{N}-\mathrm{H}), 1198$ broad $\left(\mathrm{C}-\mathrm{O}-\mathrm{C}\right.$ and $\left.\mathrm{SO}_{3}\right), 922$ $\left(=\mathrm{CH}_{2}\right)$.

Gly-AAla-OPrg.Tos $71 \%$ global yield; $\mathrm{mp}=141-$ $143.5{ }^{\circ} \mathrm{C}$ with decomposition; ${ }^{1} \mathrm{H}$ NMR $\delta 9.98(\mathrm{~s}, 1 \mathrm{H}, \mathrm{NH})$, $8.05\left(\mathrm{~s}, 3 \mathrm{H}, \mathrm{NH}_{3}^{+}\right), 6.36\left(\mathrm{~s}, 1 \mathrm{H}, \mathrm{CH}_{\boldsymbol{A}} \mathrm{H}_{\mathrm{B}} \Delta \mathrm{Ala}\right), 5.87(\mathrm{~s}, 1 \mathrm{H}$, $\left.\mathrm{CH}_{\mathrm{A}} \boldsymbol{H}_{\boldsymbol{B}}{ }_{\Delta \mathrm{Ala}}\right), 4.89\left(\mathrm{~d}, J=2.3 \mathrm{~Hz}, 2 \mathrm{H}, \mathrm{OCH}_{2}\right), 3.81(\mathrm{~s}$, $\left.2 \mathrm{H}, \mathrm{CH}_{2 \mathrm{Gly}}\right), 3.67(\mathrm{t}, J=2.3 \mathrm{~Hz}, 1 \mathrm{H}, \equiv \mathrm{CH}) .{ }^{13} \mathrm{C} \mathrm{NMR} \delta$ $166.20\left(\boldsymbol{C}=\mathrm{O}_{\text {amid. }}\right), 162.30\left(\boldsymbol{C}=\mathrm{O}_{\text {est }}\right), 131.69(\boldsymbol{C}=), 111.49$ $\left(\boldsymbol{C H}_{2}=\right), 78.48,77.92(2 \times \boldsymbol{C} \equiv \boldsymbol{C H}), 53.34\left(\mathrm{OCH}_{2}\right), 41.10$ $\left(C_{2 \mathrm{Gly}}\right)$. HRMS (ESI) $\mathrm{m} / \mathrm{z}$ calcd for $\mathrm{C}_{8} \mathrm{H}_{11} \mathrm{~N}_{2} \mathrm{O}_{3}$ $(\mathrm{M}+\mathrm{H})^{+}$183.0764; found 183.0771. IR $\left(\mathrm{KBr}, \mathrm{cm}^{-1}\right)$ 3600-2800 broad (H-bonding), $2129 \quad(\mathrm{C} \equiv \mathrm{C}), 1732$ $\left(\mathrm{C}=\mathrm{O}_{\text {ester }}\right), 1700 \mathrm{IAB}\left(\mathrm{C}=\mathrm{O}_{\text {amid }}\right), 1638(\mathrm{C}=\mathrm{C}), 1547$ IIAB $(\mathrm{C}-\mathrm{N}$ and $\mathrm{N}-\mathrm{H}), 1178$ broad $\left(\mathrm{C}-\mathrm{O}-\mathrm{C}\right.$ and $\left.\mathrm{SO}_{3}\right), 895$ $\left(=\mathrm{CH}_{2}\right)$.

(S)Phe-_Ala-OAll-Tos $70 \%$ global yield; $\mathrm{mp}=123.5$ $125{ }^{\circ} \mathrm{C}$ with decomposition; ${ }^{1} \mathrm{H}$ NMR $\delta 9.96$ (s, $1 \mathrm{H}, \mathrm{NH}$ ),

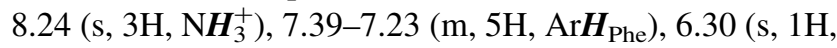
$\left.\mathrm{CH}_{\boldsymbol{A}} \mathrm{H}_{\mathrm{B}} \Delta \mathrm{Ala}\right), 6.03-5.91\left(\mathrm{~m}, 1 \mathrm{H}, \mathrm{CH}_{2}=\mathrm{CH}\right), 5.89(\mathrm{~s}, 1 \mathrm{H}$, $\left.\mathrm{CH}_{\mathrm{A}} \boldsymbol{H}_{\boldsymbol{B}} \Delta_{\Delta \mathrm{Ala}}\right), \quad 5.40-5.33\left(2 \times \mathrm{m}, \quad 1 \mathrm{H}, \quad \mathrm{CH}=\mathrm{CH}_{A} \mathrm{H}_{\mathrm{B}}\right)$, 5.30-5.25 $\left(2 \times \mathrm{m}, 1 \mathrm{H}, \mathrm{CH}=\mathrm{CH}_{\mathrm{A}} \boldsymbol{H}_{\boldsymbol{B}}\right), 4.71\left(\mathrm{~m}, 2 \mathrm{H}, \mathrm{OCH}_{2}\right)$, 4.40 (wide s, $1 \mathrm{H}, \mathrm{CH}_{\mathrm{Phe}}$ ), $3.10(\mathrm{dd}, \mathrm{J}=13.9,6.2 \mathrm{~Hz}, 1 \mathrm{H}$, $\mathrm{ABX}$ system $\mathrm{CH}_{\boldsymbol{A}} \mathrm{H}_{\mathrm{B}}$ Phe), $3.00(\mathrm{dd}, \mathrm{J}=13.9,7.8 \mathrm{~Hz}, 1 \mathrm{H}$, $\mathrm{ABX}$ system $\mathrm{CH}_{\mathrm{A}} \boldsymbol{H}_{\boldsymbol{B}}$ Phe $) .{ }^{13} \mathrm{C}$ NMR $\delta 168.15\left(\boldsymbol{C}=\mathrm{O}_{\text {amid. }}\right)$, $162.57\left(\boldsymbol{C}=\mathrm{O}_{\text {est. }}\right), \quad 134.59 \quad\left(\boldsymbol{C}_{\mathrm{Ar}_{\mathrm{Phe}}}\right), 132.08 \quad\left(\boldsymbol{C H}=_{\mathrm{All}}\right)$, $131.90(\boldsymbol{C}=), 129.55,128.59,127.31\left(3 \times \boldsymbol{C A r}_{\mathrm{Phe}}\right), 118.39$ $\left(\boldsymbol{C H}_{2}={ }_{\mathrm{All}}\right), 111.71 \quad\left(\boldsymbol{C H}_{2}={ }_{\Delta \mathrm{Ala}}\right), 65.92\left(\mathrm{OCH}_{2}\right), \quad 53.70$ $\left(\boldsymbol{C} \mathrm{H}_{\text {Phe }}\right)$, $37.09\left(\boldsymbol{C H}_{2 \mathrm{Phe}}\right)$. HRMS (ESI) $\mathrm{m} / \mathrm{z}$ calcd for $\mathrm{C}_{15} \mathrm{H}_{19} \mathrm{~N}_{2} \mathrm{O}_{3}(\mathrm{M}+\mathrm{H})^{+}$275.1390; found 275.1381. IR (KBr, $\mathrm{cm}^{-1}$ ) 3600-2700 broad (H-bonding), $1722\left(\mathrm{C}=\mathrm{O}_{\text {ester }}\right), 1699$ IAB $\left(\mathrm{C}=\mathrm{O}_{\text {amid }}, 1637(\mathrm{C}=\mathrm{C}), 1527\right.$ IIAB $(\mathrm{C}-\mathrm{N}$ and $\mathrm{N}-\mathrm{H})$, 1231-1176 $\left(\mathrm{C}-\mathrm{O}-\mathrm{C}\right.$ and $\left.\mathrm{SO}_{3}\right), 947\left(=\mathrm{CH}_{2}\right)$.

(S)Phe-AAla-OPrg-Tos $65 \%$ global yield; $\mathrm{mp}=$ 170-172 ${ }^{\circ} \mathrm{C}$ with decomposition; ${ }^{1} \mathrm{H}$ NMR $\delta 10.02$ (s, $1 \mathrm{H}$ $\mathrm{NH}), 8.24$ (s, 3H, NH $\left.\boldsymbol{H}_{3}^{+}\right), 7.39-7.24\left(\mathrm{~m}, 5 \mathrm{H}, \operatorname{Ar} \boldsymbol{H}_{\mathrm{Phe}}\right), 6.30$ $\left(\mathrm{s}, 1 \mathrm{H}, \mathrm{CH}_{\boldsymbol{A}} \mathrm{H}_{\mathrm{B}} \Delta \mathrm{Ala}\right), 5.89\left(\mathrm{~s}, 1 \mathrm{H}, \mathrm{CH}_{\mathrm{A}} \boldsymbol{H}_{\boldsymbol{B}} \Delta \mathrm{Ala}\right), 4.87(\mathrm{~d}$, $\mathrm{J}=2.3 \mathrm{~Hz}, 2 \mathrm{H}, \mathrm{OCH}_{2}$ ), 4.38 (wide s, $1 \mathrm{H}, \mathrm{CH}_{\mathrm{Phe}}$ ), 3.68 (t, $\mathrm{J}=2.3 \mathrm{~Hz}, 1 \mathrm{H}, \equiv \mathrm{CH}), 3.11(\mathrm{dd}, \mathrm{J}=13.9,6.0 \mathrm{~Hz}, 1 \mathrm{H}$, $\mathrm{ABX}$ system $\left.\mathrm{CH}_{\mathbf{A}} \mathrm{H}_{\mathrm{B} \text { Phe }}\right), 3.00(\mathrm{dd}, \mathrm{J}=13.9,7.8 \mathrm{~Hz}, 1 \mathrm{H}$, $\mathrm{ABX}$ system $\mathrm{CH}_{\mathrm{A}} \boldsymbol{H}_{\boldsymbol{B}}$ Phe $) .{ }^{13} \mathrm{C}$ NMR $\delta 168.20\left(\boldsymbol{C}=\mathrm{O}_{\text {amid. }}\right)$, $162.22\left(\boldsymbol{C}=\mathrm{O}_{\text {est. }}\right), 134.58\left(\boldsymbol{C}\right.$ Ar $\left._{\text {Phe }}\right), 131.58(\boldsymbol{C}=), 129.56$, 128.61, $127.34\left(3 \times \boldsymbol{C}\right.$ Ar $\left._{\text {Phe }}\right), 112.52\left(\boldsymbol{C H}_{2}=\Delta \mathrm{Ala}\right), 78.48$, 
$77.89(2 \times \boldsymbol{C} \equiv \boldsymbol{C H}), 53.72\left(\boldsymbol{C H}_{\mathrm{Phe}}\right), 53.32\left(\mathrm{OCH}_{2}\right), 37.07$ $\left(\boldsymbol{C H}_{2 \mathrm{Phe}}\right)$. HRMS (ESI) $\mathrm{m} / \mathrm{z}$ calcd for $\mathrm{C}_{15} \mathrm{H}_{17} \mathrm{~N}_{2} \mathrm{O}_{3}$ $(\mathrm{M}+\mathrm{H})^{+}$273.1234; found 273.1224. IR $\left(\mathrm{KBr}, \mathrm{cm}^{-1}\right)$ 3600-2850 broad (H-bonding), $2120 \quad(\mathrm{C} \equiv \mathrm{C}), 1745$ $\left(\mathrm{C}=\mathrm{O}_{\text {ester }}\right), 1699$ IAB $\left(\mathrm{C}=\mathrm{O}_{\text {amid }}\right), 1632(\mathrm{C}=\mathrm{C}), 1517$ IIAB $(\mathrm{C}-\mathrm{N}$ and $\mathrm{N}-\mathrm{H}), 1227-1168$ broad $\left(\mathrm{C}-\mathrm{O}-\mathrm{C}\right.$ and $\left.\mathrm{SO}_{3}\right)$.

\section{Synthesis of allyl and propargyl esters of dipeptides containing $(Z)$-dehydrophenylalanine}

Boc-Gly- $\Delta^{\mathrm{Z}}$ Phe $0.320 \mathrm{~g}(1.0 \mathrm{mmol})$ was dissolved in $5 \mathrm{~mL}$ DMF, and $\mathrm{Cs}_{2} \mathrm{CO}_{3} 0.163 \mathrm{~g}(0.5 \mathrm{mmol})$ was added. Mixture was stirred for $3 \mathrm{~h}$, and allyl bromide $0.856 \mathrm{~mL}(10 \mathrm{mmol})$ or propargyl bromide $1.114 \mathrm{~mL}(10 \mathrm{mmol})$ was added dropwise over $15 \mathrm{~min}$. The reaction was continued for $12 \mathrm{~h}$ stirring at room temperature. Further steps of synthesis were done according to procedure described for allyl and propargyl esters of Boc-Gly- $\Delta$ Ala. The deprotection reaction of amine group was performed without addition of anisole and $p$-toluenesulfonic acid.

Gly- $\Delta^{Z}$ Phe-OAll.TFA $88 \%$ global yield; $\mathrm{mp}=137-$ $138.5{ }^{\circ} \mathrm{C}$ with decomposition; ${ }^{1} \mathrm{H}$ NMR $\delta 10.19$ (s, $1 \mathrm{H}$, $\mathrm{NH}), 8.19$ (s, 3H, NH $\left.\boldsymbol{H}_{3}^{+}\right), 7.78-7.41$ (m, 5H, Ar $\left.\boldsymbol{H}_{\Delta \mathrm{Phe}}\right), 7.39$ $\left(\mathrm{s}, 1 \mathrm{H}, \mathrm{CH}_{\Delta \mathrm{Phe}}\right), 6.08-5.90\left(\mathrm{~m}, 1 \mathrm{H}, \mathrm{CH}_{2}=\mathrm{CH}\right), 5.43-5.34$ $\left(2 \times \mathrm{m}, \quad 1 \mathrm{H}, \quad \mathrm{CH}=\mathrm{CH}_{\boldsymbol{A}} \mathrm{H}_{\mathrm{B}}\right), \quad 5.30-5.23 \quad(2 \times \mathrm{m}, \quad 1 \mathrm{H}$, $\left.\mathrm{H}=\mathrm{CH}_{\mathrm{A}} \boldsymbol{H}_{\boldsymbol{B}}\right), 4.69\left(\mathrm{~m}, 2 \mathrm{H}, \mathrm{OCH}_{2}\right), 3.81$ (s, 2H, CH $\left.\boldsymbol{H}_{2 \mathrm{Gly}}\right)$. ${ }^{13} \mathrm{C}$ NMR $\delta 166.22\left(\boldsymbol{C}=\mathrm{O}_{\text {amid. }}\right), 164.08\left(\boldsymbol{C}=\mathrm{O}_{\text {est. }}\right), 133.11$, 132.87, 132.44, 130.18, 129.89, 128.79, 124.87, 117.99 (8 $\mathrm{C}$ atoms derived from (Z)-dehydrophenylalanine and allyl group), $65.54\left(\mathrm{OCH}_{2}\right), 40.38\left(\mathrm{CH}_{2 \mathrm{Gly}}\right)$, (peaks derived from TFA group are omitted for clarity). HRMS (ESI) m/z calcd for $\mathrm{C}_{14} \mathrm{H}_{17} \mathrm{~N}_{2} \mathrm{O}_{3}(\mathrm{M}+\mathrm{H})^{+}$261.1234; found 261.1229. IR $\left(\mathrm{KBr}, \mathrm{cm}^{-1}\right.$ ) 3600-2600 broad (H-bonding), 1723 $\left(\mathrm{C}=\mathrm{O}_{\text {ester }}\right), 1698$ IAB $\left(\mathrm{C}=\mathrm{O}_{\text {amid }}\right), 1625(\mathrm{C}=\mathrm{C}), 1529$ IIAB $(\mathrm{C}-\mathrm{N}$ and $\mathrm{N}-\mathrm{H}), 1201-1180(\mathrm{C}-\mathrm{O}-\mathrm{C}), 922\left(=\mathrm{CH}_{2}\right), 837$ $\left(=\mathrm{CH}_{\Delta \mathrm{Phe}}\right)$.

Gly- $\triangle^{Z}$ Phe-OPrg.TFA $92 \%$ global yield; $\mathrm{mp}=145-$ $147{ }^{\circ} \mathrm{C}$ with decomposition; ${ }^{1} \mathrm{H}$ NMR $\delta 10.21$ (s, 1H, NH), 8.20 (s, 3H, NH $\left.H_{3}^{+}\right), 7.73-7.42\left(\mathrm{~m}, 5 \mathrm{H}, \mathrm{Ar} \boldsymbol{H}_{\Delta \mathrm{Phe}}\right), 7.40$ (s, $\left.1 \mathrm{H}, \mathrm{CH}_{\Delta \mathrm{Phe}}\right), 4.84$ (d, $\left.\boldsymbol{J}=2.4 \mathrm{~Hz}, 2 \mathrm{H}, \mathrm{OCH}_{2}\right), 3.81$ (s, $\left.2 \mathrm{H}, \mathrm{CH}_{2 \mathrm{Gly}}\right), 3.64(\mathrm{t}, J=2.4 \mathrm{~Hz}, 1 \mathrm{H}, \equiv \mathrm{CH}) .{ }^{13} \mathrm{C} \mathrm{NMR} \delta$ $166.24\left(\boldsymbol{C}=\mathrm{O}_{\text {amid. }}\right), 163.72\left(\boldsymbol{C}=\mathrm{O}_{\text {est. }}\right), 133.88,132.74$, 130.27, 130.07, 128.83, 124.33 (6 $\mathrm{C}$ atoms derived from (Z)-dehydrophenylalanine), 78.29, $78.16(2 \times \boldsymbol{C} \equiv \boldsymbol{C H})$, $52.86\left(\mathrm{OCH}_{2}\right), 40.38\left(\mathrm{CH}_{2 \mathrm{Gly}}\right)$ (for clarity peaks derived from TFA group are omitted); HRMS (ESI) $\mathrm{m} / \mathrm{z}$ calcd for $\mathrm{C}_{14} \mathrm{H}_{15} \mathrm{~N}_{2} \mathrm{O}_{3}(\mathrm{M}+\mathrm{H})^{+}$259.1077; found 259.1060. IR (KBr, cm ${ }^{-1}$ ) 3600-2600 broad (H-bonding), $2132(\mathrm{C} \equiv \mathrm{C})$, $1723\left(\mathrm{C}=\mathrm{O}_{\text {ester }}\right), 1698 \mathrm{IAB}\left(\mathrm{C}=\mathrm{O}_{\text {amid }}\right), 1624(\mathrm{C}=\mathrm{C}) 1531$ IIAB (C-N and N-H), 1201-1179 (C-O-C), 837 $\left(=\mathrm{CH}_{\Delta \mathrm{Phe}}\right)$.
Efforts to synthesize dehydrodipeptide glycidyl esters

Method I Boc-Gly- $\Delta^{\mathrm{Z}} \mathrm{Phe} 0.160 \mathrm{~g} \quad(0.5 \mathrm{mmol}), \mathrm{Et}_{3} \mathrm{~N}$ $0.196 \mathrm{~mL}(1.1 \mathrm{mmol})$ and $(S)$-glycidol $0.266 \mathrm{~mL}(2.0$ mmol) were dissolved in $2.0 \mathrm{~mL}$ of acetonitrile, and TBTU (Abdelmoty et al., 1994) $0.208 \mathrm{~g}$ (0.65 mmol) was then added. Mixture was stirred at room temperature for $2.5 \mathrm{~h}$, and solvent removed under reduced pressure. The residue was dissolved in $70 \mathrm{~mL}$ of ethyl acetate and washed subsequently with: $1 \mathrm{M} \mathrm{HCl}(3 \times 5 \mathrm{~mL})$, saturated $\mathrm{KHCO}_{3}$ $(3 \times 5 \mathrm{~mL})$ and brine. Organic phase was dried over $\mathrm{MgSO}_{4}$ and filtered, and solvents were removed. We were unable to purify a mixture of products obtained using column chromatography with silica gel $60 \mathrm{H}$ (Merck) as stationary phase and various eluents. Thus, crude mixture was used in deprotection step. HRMS (ESI) indicated the presence of the desired product as a major one: $\mathrm{m} / \mathrm{z}$ calcd for $\mathrm{C}_{19} \mathrm{H}_{24} \mathrm{~N}_{2} \mathrm{O}_{6}(\mathrm{M}+\mathrm{Na})^{+}$399.1526; found 399.1529.

Method II iso-butyl chloroformate $0.066 \mathrm{~mL}(0.5 \mathrm{mmol})$ was added to solution of Boc-Gly- $\Delta^{\mathrm{Z}}$ Phe $0.160 \mathrm{~g}(0.5$ $\mathrm{mmol})$ and $\mathrm{Et}_{3} \mathrm{~N} 0.070 \mathrm{~mL}(0.5 \mathrm{mmol})$ in dichloromethane when cooling in ice bath to $-15^{\circ} \mathrm{C}$. After $1.5 \mathrm{~min}$, glycidol $0.133 \mathrm{~mL}(1.0 \mathrm{mmol})$ was added. The mixture was left to warm to room temperature, and stirring was continued for next $24 \mathrm{~h}$. Further steps of synthesis were performed according to the methodology described for Method I and afforded similar mixture of products.

\section{Deprotection of amine group}

Method I Trifluoroacetic acid $0.5 \mathrm{~mL}$ was added to solution of Boc-Gly- $\Delta^{\mathrm{Z}}$ Phe-OGdl $0.098 \mathrm{~g}(0.25 \mathrm{mmol})$ in $2 \mathrm{~mL}$ of dichloromethane. Mixture was stirred for $20 \mathrm{~min}$ at room temperature, and solvent was removed under reduced pressure. The residue was evaporated three times with $20 \mathrm{~mL}$ of dichloromethane and $20 \mathrm{~mL}$ of diethyl ether to remove the excess of trifluoroacetic acid. Mixture of products was obtained as oily residue. HRMS (ESI) indicated the presence of the two major products-desired glycidol ester (Gly- $\left.\Delta^{\mathrm{Z}} \mathrm{Phe}-\mathrm{OGdl}(\mathrm{S})\right)$ and the product of oxirane ring opening-Gly- $\Delta{ }^{\mathrm{Z}} \mathrm{Phe}-\mathrm{OCH}_{2} \mathrm{CH}(\mathrm{OH}) \mathrm{CH}_{2} \mathrm{OH}$ : $\mathrm{m} / \mathrm{z}$ calcd for $\mathrm{C}_{14} \mathrm{H}_{17} \mathrm{~N}_{2} \mathrm{O}_{4}(\mathrm{M}+\mathrm{H})^{+} 277.1183$ and $\mathrm{C}_{14} \mathrm{H}_{19} \mathrm{~N}_{2} \mathrm{O}_{5}(\mathrm{M}+\mathrm{H})^{+}$295.1288; found 277.1164 and 295.1266, respectively.

Method II HCl in methanol $(\sim 3.8 \mathrm{M})$ solution was prepared by bubbling dry $\mathrm{HCl}$ gas through methanol for $1 \mathrm{~h}$ at $0{ }^{\circ} \mathrm{C}$. Crude Boc-Gly- $\Delta^{\mathrm{Z}}$ Phe-OGdl(S) $0.129 \mathrm{~g}(0.34$ mmol) was dissolved in methanol $(1.2 \mathrm{~mL})$, and $\mathrm{HCl}-$ methanol solution was added $(1.3 \mathrm{~mL})$. After $1 \mathrm{~h}$ at room temperature, solvent was evaporated under reduced pressure. The oil residue was evaporated three times with $5 \mathrm{~mL}$ of dichloromethane. Product was crystallized from mixture 
of isopropanol/diethyl ether/hexane (2:1), filtered and dried in vacuo.

In that manner, $\mathrm{Gly}-\Delta^{\mathrm{Z}} \mathrm{Phe}-\mathrm{OCH}_{2} \mathrm{CH}(\mathrm{OH}) \mathrm{CH}_{2} \mathrm{Cl} \cdot \mathrm{HCl}$ was obtained as a white solid in $50 \%$ yield (deprotection): $\mathrm{mp}=178-180{ }^{\circ} \mathrm{C}$ decomposition; ${ }^{1} \mathrm{H}$ NMR $\delta 10.28(\mathrm{~s}$, $1 \mathrm{H}, \mathrm{NH}), 8.30\left(\mathrm{~s}, 3 \mathrm{H}, \mathrm{NH}_{3}^{+}\right), 7.74-7.40(2 \times \mathrm{m}, 2 \mathrm{H}$ and $4 \mathrm{H}, \quad \operatorname{Ar}_{\Delta(\mathrm{Z}) \mathrm{Phe}}$ overlapped with $\left.\mathrm{CH}_{\Delta(\mathrm{Z}) \mathrm{Phe}}\right), 5.67$ (d, $J=5.1 \mathrm{~Hz}, 1 \mathrm{H}, \mathrm{OH}), 4.18(\mathrm{dd}, J=11.1,5.2 \mathrm{~Hz}, 1 \mathrm{H})$, $4.13(\mathrm{dd}, J=11.1,5.7 \mathrm{~Hz}, 1 \mathrm{H}), 4.05-3.97(\mathrm{~m}, 1 \mathrm{H}$, $\mathrm{CHOH}$ ), 3.79 (s, 2H, $\mathrm{CH}_{2 \mathrm{Gly}}$ ), 3.73 (dd, $J=11.3,4.8 \mathrm{~Hz}$, $1 \mathrm{H}), 3.66(\mathrm{dd}, J=11.3,5.5 \mathrm{~Hz}, 1 \mathrm{H})$. Four dd at 4.18, 4.13, 3.73, $3.66 \mathrm{ppm}$ derived from two $\mathrm{CH}_{2}$ groups which are present at $\mathrm{OCH}_{2} \mathrm{CH}(\mathrm{OH}) \mathrm{CH}_{2} \mathrm{Cl}$ part of the molecule. ${ }^{13} \mathrm{C}$ NMR $\delta 166.21 \quad\left(\boldsymbol{C}=\mathrm{O}_{\text {amid. }}\right), 164.22 \quad\left(\boldsymbol{C}=\mathrm{O}_{\text {est. }}\right), 133.44$, 132.91, 130.24, 129.87, 128.78, 124.62 (6 C atoms derived from (Z)-dehydrophenylalanine), 67.94, 66.14, 46.56 $\left(\mathrm{OCH}_{2} \mathrm{CH}(\mathrm{OH}) \mathrm{CH}_{2} \mathrm{Cl}\right), 40.37\left(\boldsymbol{C H}_{2 \mathrm{Gly}}\right)$. HRMS (ESI) m/z calcd for $\mathrm{C}_{14} \mathrm{H}_{18} \mathrm{ClN}_{2} \mathrm{O}_{4}(\mathrm{M}+\mathrm{H})^{+}$313.0950; found 313.0950; intensity of ions: $313.0950 \mathrm{I}=100 \%$; $315.0926 \mathrm{I}=34.2 \%$ (chlorine isotopes). $\mathrm{IR}\left(\mathrm{KBr}, \mathrm{cm}^{-1}\right)$ 3600-2550 broad (H-bonding), $1706\left(\mathrm{C}=\mathrm{O}_{\text {ester }}\right), 1680$ IAB $\left(\mathrm{C}=\mathrm{O}_{\text {amid }}\right), 1636(\mathrm{C}=\mathrm{C}), 1541$ IIAB $(\mathrm{C}-\mathrm{N}$ and $\mathrm{N}-\mathrm{H}), 841$ $\left(=\mathrm{CH}_{\Delta \mathrm{Phe}}\right)$.

\section{Enzymatic studies}

Cathepsin $\mathrm{C}$ was isolated from bovine spleen by modified method of McDonald et al. (1972). The $\mathrm{K}_{\mathrm{M}}$ value of $2.3 \mathrm{mM}$ for the enzyme was measured using synthetic substrate-glycine- $L$-phenylalanine- $p$-nitroanilide (Gly- $L$ Phe- $p$ NA). Purity of the enzyme was confirmed by electrophoresis.

\section{Inhibitory studies}

Cathepsin $\mathrm{C}$ was activated for $0.5 \mathrm{~h}$ in a water bath at $37{ }^{\circ} \mathrm{C}$ in $1 \% \mathrm{NaCl}$ solution containing $1 \mathrm{mM}$ EDTA-Na and $5 \mathrm{mM}$ 2-mercaptoethanol. The enzymatic reaction was carried out at $37^{\circ} \mathrm{C}$ in $100 \mathrm{mM}$ acetate buffer, pH 5.0, containing $1 \mathrm{mM}$ EDTA-Na, $1 \mathrm{mM}$ DTT and $30 \mathrm{mM}$ $\mathrm{NaCl}$ (all final concentrations). The progress of the reaction was monitored spectrophotometrically (UV-Vis spectrophotometer Cintra 303) at a wavelength of $405 \mathrm{~nm}$ against a control sample containing no enzyme. Attempting mixture contained: solution of the synthetic substrate Gly$L$-Phe- $p \mathrm{NA}$ in acetate buffer at $\mathrm{pH} 5$ containing $1 \mathrm{mM}$ EDTA-Na, $1 \mathrm{mM}$ DTT, $30 \mathrm{mM} \mathrm{NaCl}$ (substrate concentration: $2.7-0.01 \mathrm{mM}$ - final concentration), the solution of inhibitor in reaction buffer (concentration of compound depended on inhibitory potential), and enzyme.

Kinetic constants $\mathrm{K}_{\mathrm{M}}, \mathrm{V}_{\max }$ and $\mathrm{K}_{\mathrm{i}}$ and type of inhibition were determined by using Lineweaver-Burk, Dixon, Hanes-Woolf and half-inhibitory concentration methods using the computer program provided kindly by dr Józef Hurek (University of Opole). The $K_{\mathrm{i}}$ values presented in the Table 1 are the average ones calculated by using all these methods. All measurements were taken in a three repetitions.

\section{Molecular modeling}

The structures of studied dehydropeptides were optimized in Gaussian09 program at the B3LYP/6-311 g (d,p) level (Frisch et al., 2004) in gas phase with using Merz-SinghKollman scheme (Besler et al., 1990) to the determination of the atomic charges. The calculations of the docking process were performed using AutoDock program (Morris et al., 2009). The starting geometry and charges of the dehydropeptides were taken from the ab initio calculations. The structure of cathepsin $\mathrm{C}$ was extracted from the structure of human dipeptidyl peptidase I deposited EC 3.4.14 in Protein Data Bank (Turk et al., 2001). Structure of the enzyme has been protonated on the $\mathrm{H}++$ server (Myers et al., 2006) at $\mathrm{pH}=5.7$, and also charges of all enzymatic atoms have been assignment on this server. During the docking process, main chain of the dehydropeptide was fixed, whereas side chains and the terminal groups were left as flexible. The coordinates of the $\mathrm{SH}$ proton from the Cys 234 were taken as a grid center in the docking process. In the simulation, docking process was performed 100 times. Analysis of the obtained results has been performed by using AutoDock Tools (Morris et al., 2009).

\section{Results and discussion}

Cathepsin C (EC 3.4.14.1) is a lysosomal cysteine protease expressed in majority of mammalian tissues and is primarily responsible for activation of serine proteases in inflammatory and immune cells (Reiser et al., 2010). It sequentially removes dipeptides from the $N$-termini of protein and peptide substrates (Lindley, 1972; Poręba et al., 2014). Increasing evidence of the key role of DPPI in various diseases, such as sepsis, asthma, Duchenne muscular dystrophy, rheumatoid arthritis, basal cell carcinomas, chronic obstructive pulmonary disease and other inflammatory disorders (Guay et al., 2010; Laine and Busch-Petersen, 2010), stimulates interest in this enzyme as the possible medicinal target.

Dehydropeptides appear to be weak inhibitors of the enzyme (Latajka et al., 2006, 2008). In this paper, we synthesized series of structurally variable esters of gly$\mathrm{cyl}^{\mathrm{Z}}$ dehydrophenylalanine and its analogs. We speculated that the possible binding of the aromatic part of the inhibitor within S2 pocket of the enzyme might result in 
Table 1 Inhibitory constants of the studied dehydrodipeptides toward cathepsin C

\begin{tabular}{|c|c|c|c|}
\hline Compound & $K_{\mathrm{i}}(\mu \mathrm{M})$ & Compound & $\mathrm{K}_{\mathrm{i}}(\mu \mathrm{M})$ \\
\hline (S)Phe-AlaOMe-Tos & $416 \pm 10$ & & \\
\hline Gly- $\Delta \mathrm{AlaOMe} \cdot \mathrm{Tos}$ & NI & $(S)$ Phe- $\Delta$ AlaOMe $\cdot$ Tos & $64 \pm 3$ \\
\hline$(S) \mathrm{Phe}-\Delta \mathrm{AlaOEt} \cdot \mathrm{Tos}$ & $84 \pm 4$ & (S)Phe- $\Delta$ AlaOPr ${ }^{i} \cdot \mathrm{Tos}$ & $171 \pm 8$ \\
\hline Gly- $\Delta$ AlaOAll·Tos & $460 \pm 20$ & Gly- ${ }^{\mathrm{z}} \Delta \mathrm{PheOAll \cdot TFA}$ & $13 \pm 1$ \\
\hline$(S)$ Phe- $\Delta$ AlaOAll $\cdot$ Tos & $17 \pm 1$ & & \\
\hline Gly- $\Delta$ AlaOPrg $\cdot$ Tos & $320 \pm 20$ & Gly- ${ }^{\mathrm{z}} \Delta$ PheOPrg $\cdot$ TFA & $33 \pm 2$ \\
\hline (S)Phe- $\Delta$ AlaOPrg.Tos & $86 \pm 4$ & Gly- $\Delta^{\mathrm{Z}} \mathrm{PheO}-\mathrm{CH}_{2} \mathrm{CH}(\mathrm{OH}) \mathrm{CH}_{2} \mathrm{Cl} \cdot \mathrm{HCl}$ & $5.5 \pm 0.5$ \\
\hline
\end{tabular}

NI-no inhibition up to $1245 \mathrm{mM}$

reaction between active ester (allyl, propargyl or glycidyl) with thiol moiety of the active-site cysteine. Unfortunately, obtained compounds exerted moderate inhibitory activity acting as competitive inhibitors. More likely this results from different than expected binding mode of these compounds.

\section{Synthesis of inhibitors}

Esters of dehydropeptides have been synthesized using classical methods of peptide chemistry. The synthetic schemes are outlined in Figs. 1 and 2. As seen from the figures for each group of esters, specific method of their preparation should be elaborated. Direct esterification of Boc-Gly- $\Delta$ Ala with DMTMM (Kunishima et al., 1999) as coupling agent gave non-satisfactory results $(30 \%$ of yield). Far better results for esterification of Boc-Gly- $\Delta$ Ala were obtained via nucleophilic substitution of alkyl halides with dipeptide cesium salts (Fig. 1). This method gives product with yield $91 \%$. Glycidyl esters seem to be more interesting as inhibitors of cathepsin since they posses oxirane ring, which is known to react preferably with the enzyme active-site cysteine. In order to prepare these esters, two standard methods, both basing on the activation of carboxylic moiety, have been elaborated (Fig. 2). Unfortunately, the reaction afforded inseparable mixture of glycidyl ester and some products of oxirane ring opening. Efforts to remove Boc protection by trifluoroacetic acid were unsuccessful and gave even more complex mixture of products, whereas using hydrogen chloride in methanol we were able to isolate 3-chloro-2-hydroxypropyl ester of BocGly- $\Delta^{\mathrm{Z}} \mathrm{Phe}$.

\section{Inhibitory studies}

Inhibitory activities of the synthesized esters are collected in Table 1 and compared to action of methyl $L$-phenylalanyl- $L$ -

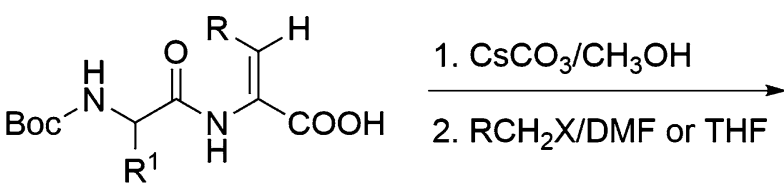<smiles>[R]C=C(NC(=O)C([R])NC(=O)OCc1ccccc1)C(=O)OC[X]</smiles>

$$
\begin{aligned}
& \text { BocGly- } \Delta \text { AlaOH: } \mathrm{R}=\mathrm{H}, \mathrm{R}^{1}=\mathrm{H} \\
& \text { BocGly- } \Delta^{(\mathrm{Z})} \text { PheOH: } \mathrm{R}=\mathrm{C}_{6} \mathrm{H}_{5}, \mathrm{R}^{1}=\mathrm{H} \\
& \text { Boc(S)Phe- } \triangle \text { AlaOH: } \mathrm{R}=\mathrm{H}, \mathrm{R}^{1}=\mathrm{CH}_{2} \mathrm{C}_{6} \mathrm{H}_{5}
\end{aligned}
$$

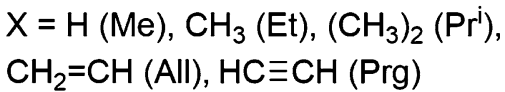

TFA/Tos<smiles>[X]COC(=O)C(NC(=O)C([R])[NH3+])=C([R])[R]</smiles>

Fig. 1 Synthesis of dehydrodipeptide methyl, ethyl, isopropyl, allyl and propargyl esters 


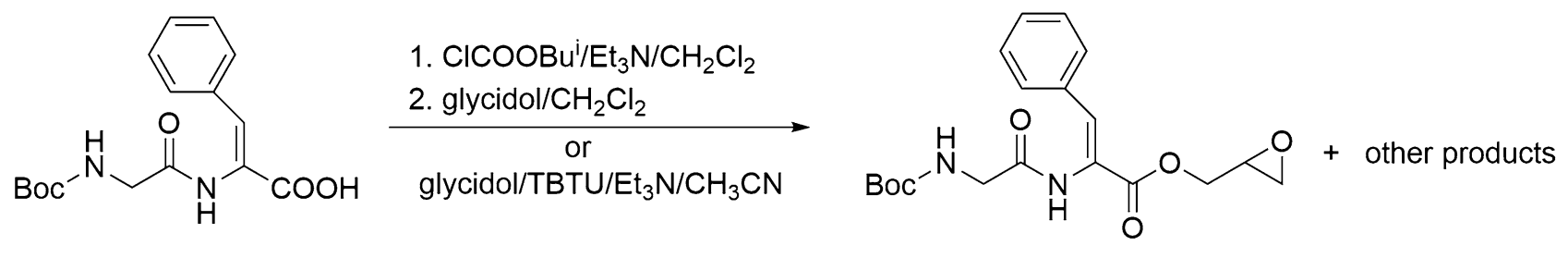

\section{BocGly- $\Delta^{(\mathrm{Z})} \mathrm{PheOGdl}$}

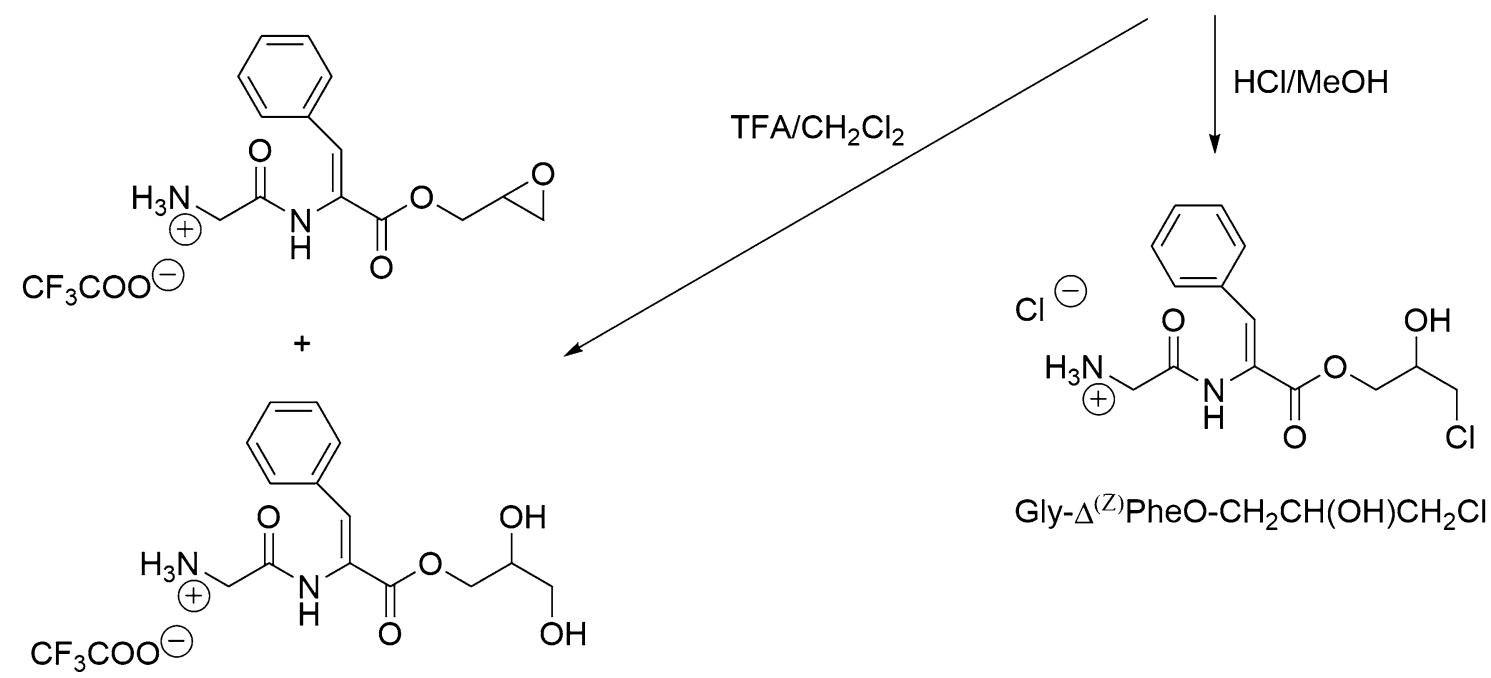

Fig. 2 Synthesis of dehydrodipeptide glycidyl ester

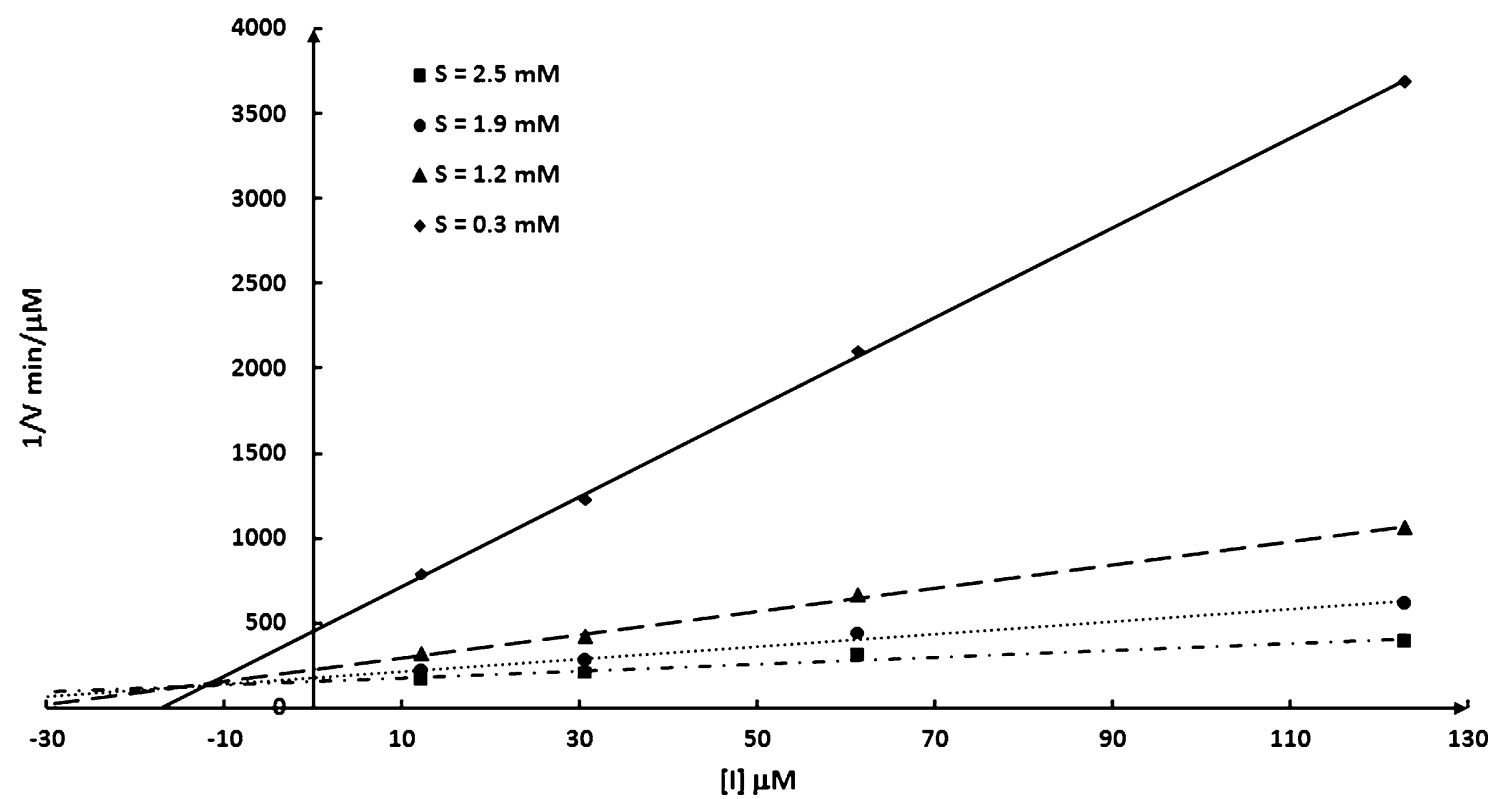

Fig. 3 Dixon plot for the hydrolysis Gly-Phe-p-NA by bovine cathepsin C versus increasing concentration of Gly- ${ }^{\mathrm{Z}} \Delta \mathrm{PheOA}$ All

alaninate (Phe-AlaOMe). All the compounds appeared to be competitive inhibitors, as shown in Fig. 3 for Gly- ${ }^{\mathrm{Z}} \Delta$ PheOAll trifluoroacetate as a representative example. The most active appeared to be $\mathrm{Gly}-\Delta^{\mathrm{Z}} \mathrm{Phe}-\mathrm{OCH}_{2} \mathrm{CH}(\mathrm{OH}) \mathrm{CH}_{2} \mathrm{Cl} \cdot \mathrm{HCl}$, Gly- ${ }^{\mathrm{Z}} \Delta$ PheOAll-TFA and $(S)$ Phe- $\Delta$ AlaOAll-Tos, which inhibitory constants were in micromolar range. Quite 

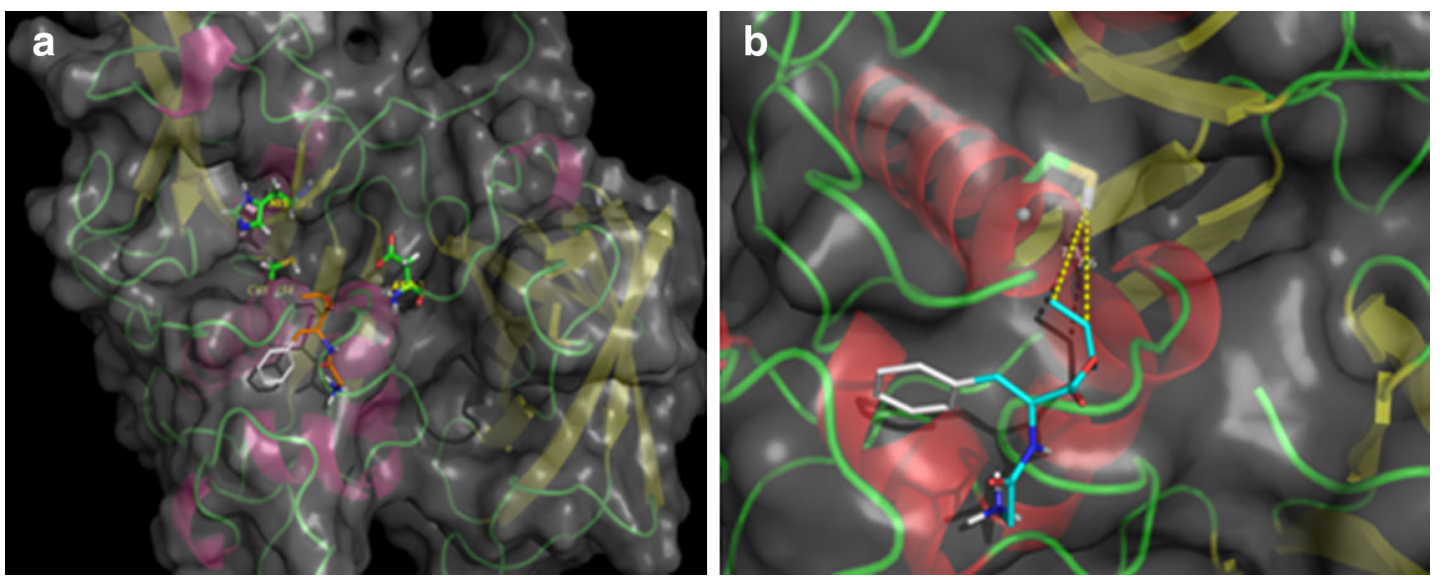

Fig. 4 a Most probable binding mode of Gly- ${ }^{\mathrm{z}} \Delta \mathrm{PheOAll}$ by cathepsin $\mathrm{C}$ and found by molecular modeling. Catalytic triad is shown in green, whereas inhibitor in white and gold. b Distance of allylic group of inhibitor from thiol moiety of active-site cysteine

interesting, six of the peptides-Gly- ${ }^{\mathrm{Z}} \Delta \mathrm{PheOPrg} \cdot \mathrm{TFA}$, Phe$\Delta$ AlaOMe-Tos, Phe- $\Delta$ AlaOEt-Tos, (S)Phe- $\Delta$ AlaOPr $^{i} \cdot$ Tos, (S)Phe- $\Delta$ AlaOAll-Tos and (S)Phe- $\Delta$ AlaOPrg.Tos-inhibit cathepsin $\mathrm{C}$ according to slow-binding mechanism. This mechanism is of B type and considers conformational rearrangement of inhibitor after binding to the enzyme (Pawełczak and Hurek, 2014). From the data shown in Table 1, it is also not possible to derive clear-cut structureactivity relationship. Contrary to recent studies on the structural requirements for the specific substrates for cathepsin C (Poręba et al., 2014), introduction of $N$-terminal phenylalanine into peptide chain results in elevation of affinity of Phe- $\Delta$ AlaOMe-Tos if compared with Gly$\Delta \mathrm{AlaOMe} \cdot \mathrm{Tos}$. This suggests that both dipeptide and dehydrodipeptide esters are bound differently than synthetic substrate of this enzyme.

Therefore, simple studies on their presumable binding using AutoDock program had been undertaken.

\section{Molecular modeling}

Simple molecular modeling using AutoDock has shown that dehydrodipeptide esters are bound at the surface of the enzyme in a non-typical manner. Their phenyl rings are not, as expected, submerged in the cathepsin $\mathrm{C}$ cavity responsible for binding aromatic fragments of the substrates and inhibitors but are rather placed at the surface of the enzyme. The most probable binding mode of Gly ${ }^{\mathrm{z}} \Delta$ PheOAll is shown in Fig. 4. As seen from this figure, allylic double bond of the inhibitor, albeit directed toward cathepsin $\mathrm{C}$ active-site cysteine 234 , is too far away from thiol moiety (7-9 ̊) to form a covalent adduct. This non-typical pattern of binding of dehydrodipeptide esters found from calculations well explains moderate inhibitory activity of these compounds.

\section{Conclusions}

Synthesis of esters of dehydropeptides is not an easy task and requires the choice of specific method tailored to each case. Esters of dehydrodipeptides containing C-terminal dehydroalanine or (Z)-dehydrophenylalanine appeared to be moderate or weak inhibitors of cathepsin C. As suggested by molecular modeling, they are bound rather on the surface of the enzyme than inside of the binding cavities of the enzyme.

Acknowledgments These studies were supported by Wroclaw Research Centre EIT+ under the Project "Biotechnologies and advanced medical technologies"-BioMed (POIG.01.01.02-02-003/ 08)—financed from the European Regional Development Fund (Operational Programme Innovative Economy, 1.1.2). We would like to thank Dr inż. Bożena Frąckowiak-Wojtasek for the HRMS analyses. Bartosz Oszywa and Paweł Lenartowicz are recipients of PhD fellowships from a Project funded by the European Social Found.

Open Access This article is distributed under the terms of the Creative Commons Attribution 4.0 International License (http:// creativecommons.org/licenses/by/4.0/), which permits unrestricted use, distribution, and reproduction in any medium, provided you give appropriate credit to the original author(s) and the source, provide a link to the Creative Commons license, and indicate if changes were made.

\section{References}

Abdelmoty I, Albericio F, Carpino LA, Foxman BM, Kates SA (1994) Structural studies of reagents for peptide bond formation: crystal and molecular structures of HBTU and HATU. Lett Pept Sci 1:57-67

Andre F, Pinet E (1997) Tentoxin: structure-activity relationship. Application to the study of its action on chloroplast ATPsynthase. Comptes Rend Seances Biol Fil 191:401-432

Battilani P, Gualla A, Dall'Asta C, Pellacani C, Galaverna G, Giorni P, Caglieri A, Tagliaferri S, Pietri A, Dossena A, Spadaro D, 
Marchelli R, Gullino ML, Costa LG (2011) Phomopsins: an overview of phytopathological and chemical aspects, toxicity, analysis and occurrence. World Mycotoxin J 4:345-359

Besler BH, Merz KM Jr, Kollman PA (1990) Atomic charges derived from semiempirical methods. J Comput Chem 11:431-439

Christenson SD, Liu W, Toney B, Shen B (2003) A novel 4-ethylideneimidazole-5-one-containing tyrosine aminomutase in enediyne antitumor antibiotic C-1027 biosynthesis. J Am Chem Soc 125:6062-6063

Cossec B, Cosnier F, Burgart M (2008) Methyl mercapturate synthesis: an efficient, convenient and simple method. Molecules 13:2394-2407

Ferreira PM, Maia HLS, Monteiro LS, Sacramento J (2001) Michael addition of thiols, carbon nucleophiles and amines to dehydroamino acid and dehydropeptide derivatives. J Chem Soc Perkin Trans 1:3167-3173

Frisch MJ, Trucks GW, Schlegel HB, Scuseria GE, Robb MA, Cheeseman JR, Montgomery, Jr. JA, Vreven T, Kudin KN, Burant JC, Millam JM, Iyengar SS, Tomasi J, Barone V, Mennucci M, Cossi M, Scalmani G, Rega N, Petersson PA, Nakatsuji H, Hada M, Ehara Toyota MK, Fukuda R, Hasegawa J, Ishida M, Nakajima T, Honda Y, Kitao O, Nakai H, Klene M, Li X, Knox JE, Hratchian HP, Cross JB, Adamo C, Jaramillo J, Gomperts R, Stratmann RE, Yazyev O, Austin AJ, Cammi R, Pomelli C, Ochterski JW, Ayala PY, Morokuma K, Voth GA, Salvador P, Dannenberg JJ, Zakrzewski VG, Dapprich S, Daniels AD, Strain MC, Farkas O, Malick DK, Rabuck AD, Raghavachari K, Foresman JB, Ortiz JV, Cui Q, Baboul AG, Clifford S, Cioslowski J, Stefanov BB, Liu G, Liashenko A, Piskorz P, Komaromi I, Martin RL, Fox DJ, Keith T, Al-Laham MA, Peng CY, Nanayakkara A, Challacombe M, Gill PMW, Johnson B, Chen W, Wong MW, Gonzalez C, Pople JA (2004) Gaussian 03 (Revision C.02), Gaussian, Inc., Wallingford CT

Goldman CE, Sungwook C, Shandler S, DeGrado WF (2007) Foldamers as versatile frameworks for the design and evolution of function. Nature Chem Biol 3:252-262

Guay D, Beaulieu C, Percival MD (2010) Therapeutic utility and medicinal chemistry of cathepsin C inhibitors. Curr Top Med Chem 10:708-716

Gulledge BM, Aggen JB, Huang HB, Nairn AC, Chamberlin AR (2002) The microcystins and nodularins: cyclic polypeptide inhibitors of PP1 and PP2A. Curr Med Chem 9:1991-2003

Jingfeng L, Fu L, Peng Y, Zhou L (2013) Metabolites from Alternaria fungi and their bioactivities. Molecules 18:5891-5935

Kanoh K, Kohno S, Katada J, Hayashi Y, Muramatsu M, Uno I (1999) Antitumor activity of phenylahistin in vitro and in vivo. Biosci Biotechnol Biochem 63:1130-1133

Kunishima M, Kawachi C, Iwasaki F, Terao K, Tani S (1999) Synthesis and characterization of 4-(4,6-dimethoxy-1,3,5-triazin2.yl)-4-methylmorpholinium chloride. Tetrahedron 40:5327-5330

Laine DI, Busch-Petersen J (2010) Inhibitors of cathepsin C (dipeptidyl peptidase I). Expert Opin Ther Pat 20:497-506

Latajka R, Makowski M, Jegwiński M, Pawełczak M, Koroniak H, Kafarski P (2006) Peptide p-nitrophenylanilides containing (E)- dehydrophenylalanine-synthesis, structural studies and evaluation of their activity towards cathepsin C. New J Chem 30:1009-1018

Latajka R, Jewgiński M, Makowski M, Pawełczak M, Huber T, Sewald N, Kafarski P (2008) Pentapeptides containing two dehydrophenylalanine residues-synthesis, structural studies and evaluation of their activity towards cathepsin C. J Pept Sci 14:1084-1095

Lindley H (1972) The specificity of dipeptidyl aminopeptidase I (cathepsin C) and its use in peptide sequence studies. Biochem $\mathrm{J}$ 126:683-685

Makowski M, Rzeszotarska B, Kubica Z, Pietrzyński G (1985) Synthesis of peptides with $\alpha, \beta$-dehydroamino acid II. Synthesis of tert-butyloxycarbonyldipeptides of dehydroalanine and dehydrophenylalanine. Liebigs Ann Chem 893-900

Makowski M, Pawełczak M, Latajka R, Nowak K, Kafarski P (2001) Synthesis of tetrapeptide p-nitrophenylanilides containing dehydroalanine and dehydrophenylalanine and their influence on cathepsin C activity. J Pept Sci 7:141-145

McDonald K, Callahan PX, Ellis P (1972) Preparation and specificity of dipeptidyl aminopeptidase I. In: Hirs CHW, Timasheff SN (eds) Methods Ezymol, 25B:272-281

Morris GM, Huey R, Lindstrom W, Sanner MF, Belew RK, Goodsell DS, Olson AJ (2009) Autodock4 and AutoDockTools4: automated docking with selective receptor flexiblity. J Comput Chem 30:2785-2791

Myers J, Grothaus G, Narayanan S, Onufriev A (2006) A simple clustering algorithm can be accurate enough for use in calculations of pKs in macromolecules. Proteins 63:928-938

Overy DP, Nielsen KF, Smetsgaard J (2005) Roquefortine/oxaline biosynthesis pathway metabolites in penicillium ser. Corymbifera. In planta production and implications for competitive fitness. J Chem Ecol 31:2373-2390

Pawełczak M, Hurek J (2014) Enzymatic slow-binding inhibition. Chemik 68:377-384

Poręba M, Mihelic M, Krai P, Rajkovic J, Krężel A, Pawełczak M, Klemba M, Turk D, Turk B, Latajka R, Drąg M (2014) Unnatural amino acids increase activity and specificity of synthetic substrates for human and malarial cathepsin C. Amino Acids 46:931-943

Reiser J, Adair B, Reinheckel T (2010) Specialized roles for cysteine cathepsins in health and disease. J Clin Investig 120:3421-3431

Seebeck FP, Ricardo A, Szostak JW (2011) Artificial lantipeptides from in vitro translations. Chem Commun 47:6141-6143

Turk D, Janji V, Stern I, Podobnik M, Lamba D, Dahl SW, Lauritzen C, Pedersen J, Turk V, Turk B (2001) Structure of human dipeptidyl peptidase I (cathepsin C): exclusion domain added to an endopeptidase framework creates the machine for activation of granular serine proteases. EMBO J 20:6570-6582

Willey JM, van der Donk WA (2007) Lantibiotics: peptides of diverse structure and function. Annu Rev Microbiol 61:477-501

Zimmer M (2002) Green fluorescent protein (GFP): applications, structure, and related photophysical behavior. Chem Rev 102:752-781 